\title{
NUEVOS REGISTROS FÓSILES DE OTODUS (MEGASELACHUS) CHUBUTENSIS [AMEGHINO, 1901 A 1902] PARA EL MIOCENO INFERIOR DE COSTA RICA, AMÉRICA CENTRAL
}

\author{
NEW FOSSIL RECORDS OF OTODUS (MEGASELACHUS) CHUBUTENSIS \\ [AMEGHINO, 1901 A 1902] FROM THE LOWER MIOCENE OF COSTA RICA, \\ CENTRAL AMERICA
}

\author{
César A. Laurito \\ INA, Instituto Nacional de Aprendizaje \\ Investigador asociado-Departamento de Historia Natural, Museo Nacional de Costa Rica \\ cesarlaurito@ice.co.cr
}

(Recibido: 07/01/2015; aceptado: 20/07/2015)

\begin{abstract}
Lower Miocene of Santa Teresa Formation, at the southern Nicoya Peninsula and San Miguel Formation at the southeast area of the Central Valley, in the central part of the country. Both geological formations correspond to shallow marine sequences; and those founds permit to correlate them with the Rio Banano Formation, outcropping in the Caribbean region where, is also recorded the species Otodus (Megaselachus) chubutensis [Ameghino, 1901 a 1902].

Keywords: Shark teeth, Lower Miocene, Otodontidae, Megaselachus, Neotropics.

RESUMEN: en el presente estudio se describen e identifican dos dientes fósiles de tiburón provenientes de las Formaciones Santa Teresa al sur de la Península de Nicoya y San Miguel al sureste del Valle Central, en la parte central del país. Ambas formaciones geológicas corresponden a secuencias marino someras de edad Mioceno Inferior; estos hallazgos fósiles permiten correlacionar dichas secuencias con la Formación Río Banano, aflorante en la región Caribe, donde también se registra la especie Otodus (Megaselachus) chubutensis [Ameghino, 1901 a 1902].

Palabras clave: Dientes de tiburón, Mioceno Inferior, Otodontidae, Megaselachus, Neotrópico.
\end{abstract}




\section{INTRODUCCIÓN}

El objetivo de la presente nota es dar a conocer 2 nuevos registros aislados de tiburones fósiles para el Mioceno de Costa Rica. El material se encuentra depositado en la colección paleontológica de la Escuela Centroamericana de Geología en la Universidad de Costa Rica y está constituido por los ejemplares CF-5181 procedente de la localidad de Punta Barrigona, cuyos sedimentos corresponden a la Formación Santa Teresa, aflorantes en la costa pacífica del sur de la Península de Nicoya y el CF3607 colectado por el geólogo Fernando Marín en 1991 en la localidad de Patarrá, Formación San Miguel cuyos afloramientos se ubican en el sureste de la ciudad capital, San José, en la región central del país (Fig. 1).

\section{PALEONTOLOGÍA}

Orden Lamniformes Berg, 1958

Familia Otodontidae Glückman, 1964

Género Otodus Agassiz, 1838

Subgénero Megaselachus Glückman, 1964

Otodus (Megaselachus) chubutensis (Agassiz, 1838)

Mioceno Inferior (Cappetta, 2012)

\section{Sinonimia:}

Carcharodon megalodon (Charlesworth) var. Chubutensis Ameghino en Zbyszewski \& Moitinho D’Almeida (1950), p. 327, lám. 3, fig. 59; lám. 5, figs. 70, 71 y 75 y lám. 6, figs. 115 a 119.

Carcharodon subauriculatus (Agassiz) en Purdy, 1998, p. 133, lám. 1, figs. 13 y 14.

Carcharodon subauriculatus (Agassiz) en Aguilera \& Rodrigues de Aguilera, 2004, p. 370, tabla 1, fig 2.

Carcharodon subauriculatus Agassiz, 1839 en Marsili et al., 2007, p. 36, lám. 1, fig. 1.

Megaselachus chubutensis (Amerghino, 1906) en Cook et al., 2010, p. 80, fig. 2b-c.

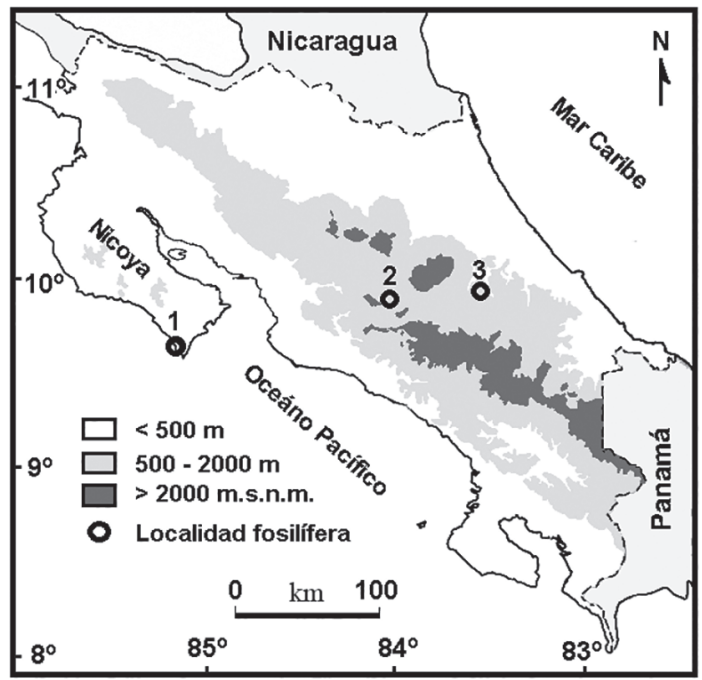

Fig. 1: Mapa de Costa Rica con la ubicación de las localidades fosilíferas portadoras de Otodus (Megaselachus) chubutensis [Ameghino, 1901 a 1902]; 1. Punta Barrigona en Mal País correspondiente a la Formación Santa Teresa, 2. San Miguel de Patarrá correspondiente a la Formación San Miguel y 3. Tres Equis correspondiente a la Formación Río Banano.

Otodus (Megaselachus) chubutensis [Ameghino 1901 a 1902] en Cappetta, 2012, p. 227.

Carcharocles chubutensis Ameghino, 1906 en Vissagi \& Godfrey, 2010, apéndices.

Carcharocles chubutensis Ameghino, 1906 en Pimiento et al., 2013, p. 161, fig. 2.

Megaselachus subauriculatus (Agassiz, 1838) en Laurito et al., 2014, p 151, fig. 4:1a-c.

Material: 2 dientes superiores, los mismos están depositados en la colección de fósiles de la Escuela Centroamericana de Geología, Universidad de Costa Rica, bajo los códigos CF5181 y CF-3607.

\section{DESCRIPCIÓN}

El CF-5181 es un diente superior casi completo (Fig. 2) y de posición mediana debido a su simetría, ello es posible deducirlo a pesar de que 
buena parte del mismo está incluido parcialmente en la matriz de calcarenita fina. Presenta una longitud total de aproximadamente $5 \mathrm{~cm}$ de alto, la corona es triangular con la cara lingual medianamente inflada y ligeramente plana en la porción central, en tanto que su cara labial al parecer es cóncava; la corona carece del ápice ya que está roto, en su parte central tiene una longitud de cerca de $2 \mathrm{~cm}$, la misma baja un $1 \mathrm{~cm}$ más en el extremo radicular visible, zona en la que no se observan cúspides accesorias pues está rota. De la raíz se observa el lóbulo lateral que en general es ancho con un margen externo muy amplio y redondeado, su porción central está marcada por un notable cuello lingual en forma de "chevron".

El CF-3607 es un diente superior (Fig. 3) y de posición lateral debido a que el mismo se encuentra ligeramente inclinado hacia la comisura, a pesar de encontrarse roto y haberse perdido su porción apical. La corona, se deduce era típicamente triangular con filos uniformemente aserrados; su cara lingual es medianamente inflada y en su base presenta un notable cuello lingual en forma de "chevron"; su cara labial es ligeramente convexa y la porción radicular se encuentra mal preservada.

Distribución paleogeográfica: Otodus (Megaselachus) chubutensis [Ameghino, 1901 a 1902] ha sido ampliamente registrada en el Mioceno Temprano de Europa (Leriche, 1926, Zbyszewski \& Moitinho D'Almeida, 1950, Menesini, 1968 y 1974, Marsili et al., 2007). También, en África, en el Mioceno Temprano de los lechos de Moghra en la depresión de Qattara en Egipto (Cook et al., 2010) y en Asia en el Mioceno Inferior de la India (Sahni \& Mehrotra, 1981).

En América del Norte se ha registrado en el Mioceno Inferior de Pollack Farm en Delaware (Purdy, 1998) y Calvert Cliffs en la Bahía de Chesapeake en Matyland (Visaggi \& Godfrey, 2010), y en las Formaciones Trent y Pungo River en Lee Creek Mine, North Carolina (Case, 1980, Purdy et al., 2001), todas en Estados Unidos; en México la especie se registra en el Mioceno
Inferior de la Península de Baja California y en la Formación El Encanto en el Estado de Veracruz (Guzmán-Guzmán et al., 2013).

En América del Sur Otodus (Megaselachus) chubutensis [Ameghino, 1901 a 1902] ha sido registrada en el Mioceno Inferior de la Formación Chilcatay en Ica, Perú (Alván et al., 2007) y de la Formación Pirabas en Brasil (Costa et al., 2009); en el Mioceno Temprano de las Formaciones Castillo y Cantaure, y la península de Paraguaná, en Venezuela (Aguilera \& Rodrigues de Aguilera, 2004, Aguilera, 2010). También, en la Formación Uitpa en el Mioceno Temprano de Colombia (Carrillo-Briceño, com. escr. 23/02/2015).

En el Caribe dicha especie ha sido descrita en el Mioceno Inferior de Cuba (Iturralde-Vinent et al., 1996) y Mioceno Medio de Puerto Rico (Nieves-Rivera et al., 2003).

En América Central ha sido recientemente registrado en el Mioceno Inferior de Panamá, en la Formación Culebra por Pimiento et al., (2013) como Carcharocles chubutensis Ameghino, 1906 y el Mioceno Inferior de Costa Rica en la localidad de Tres Equis de Turrialba, en sedimentos de la Formación Río Banano como Megaselachus subauriculatus (Agassiz, 1838) (Laurito et al., 2014).

\section{DISCUSIÓN Y CONCLUSIONES}

Los dientes descritos se caracterizan por tener coronas triangulares con filos uniformemente aserrados y en especial presentan en la base de su cara lingual el típico cuello fuertemente desarrollado tipo "chevron", lo que permite incluirlos dentro del género Otodus y descartar la posibilidad de que se trate del género Carcharodon, pues este último carece de dicho carácter lingual (sensu Glückman, 1964). Aunque los dientes del presente estudio son relativamente pequeños, si se comparan con los dientes similares de Otodus (Megaselachus) megalodon, en general son notablemente más grandes que los dientes del género Carcharodon, 

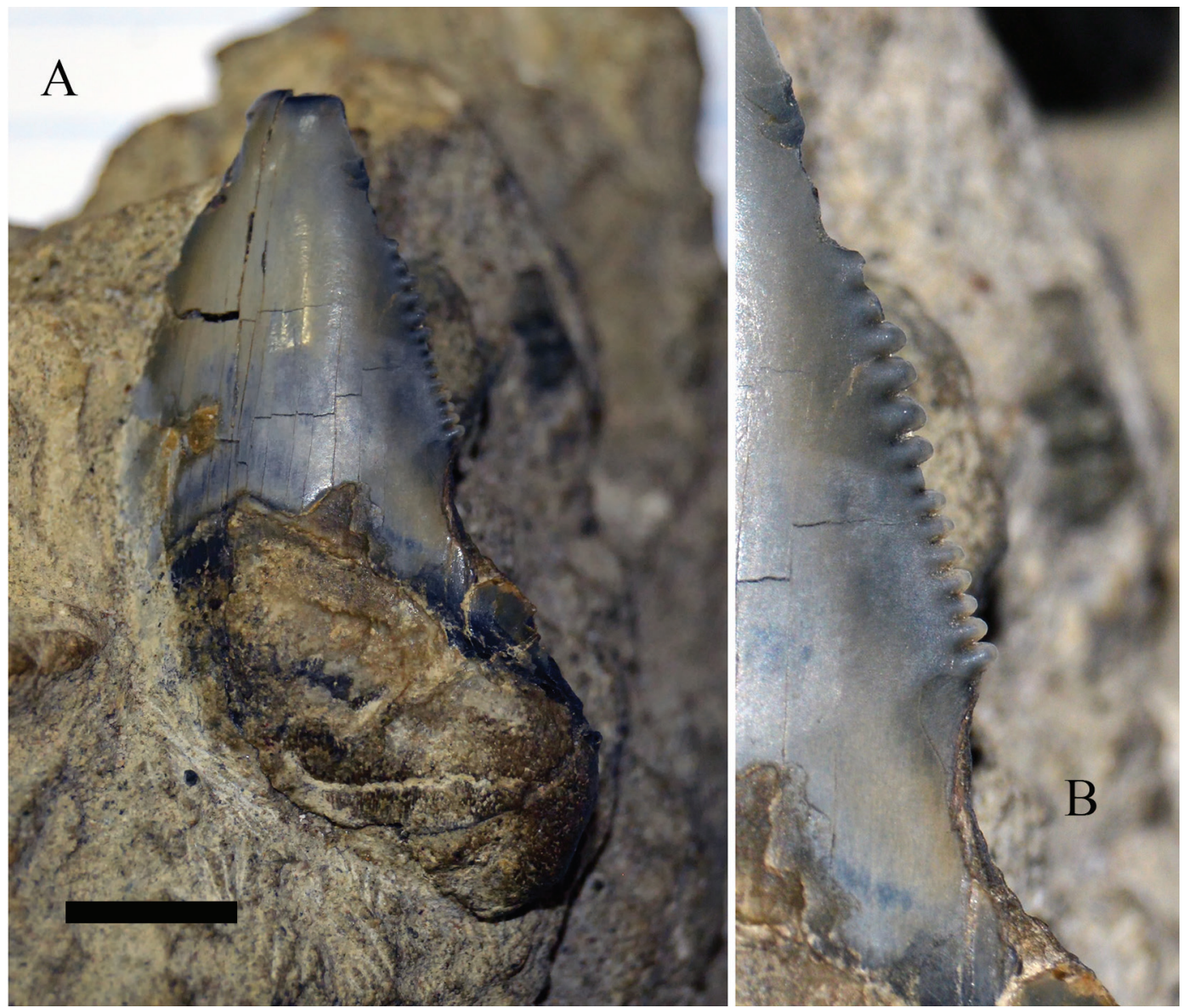

Fig. 2: A. el CF-5181 diente anterior superior de Otodus (Megaselachus) chubutensis [Ameghino, 1901 a 1902] en vista lingual, escala visual $10 \mathrm{~mm}$; B. detalle del filo uniformemente aserrado.

teniendo en cuenta lo anterior, se pueden incluir los 2 dientes dentro del subgénero Megaselachus y concluir que se trata de la especie Otodus (Megaselachus) chubutensis [Ameghino, 1901 a 1902] que en general se acepta es una cronoespecie.

La edad de los sedimentos en que se hallaron las piezas corresponden al Mioceno Temprano, tanto para el ejemplar CF-5181 procedente de la Formación Santa Teresa (cf. Mora, 1985), como para el ejemplar CF-3607 procedente de la Formación San Miguel (cf. Denyer \& Arias, 1991). Tales edades concuerdan con el rango de distribución bioestratigráfica de la especie Otodus
(Megaselachus) chubutensis [Ameghino, 1901 a 1902] de acuerdo con Cappetta (2012), rango estratigráfico que comparte con los ejemplares previamente descritos para la localidad de Tres Equis, recuperados en la Formación Río Banano (ver Laurito et al., 2014).

Por último, la distribución paleobiogeográfica de la especie Otodus (Megaselachus) chubutensis [Ameghino, 1901 a 1902], sugiere que se trataba de una especie pantropical e incluso pansubtropical, habitante de las plataformas continentales e insulares y probablemente fue un poderoso nadador oceánico. 


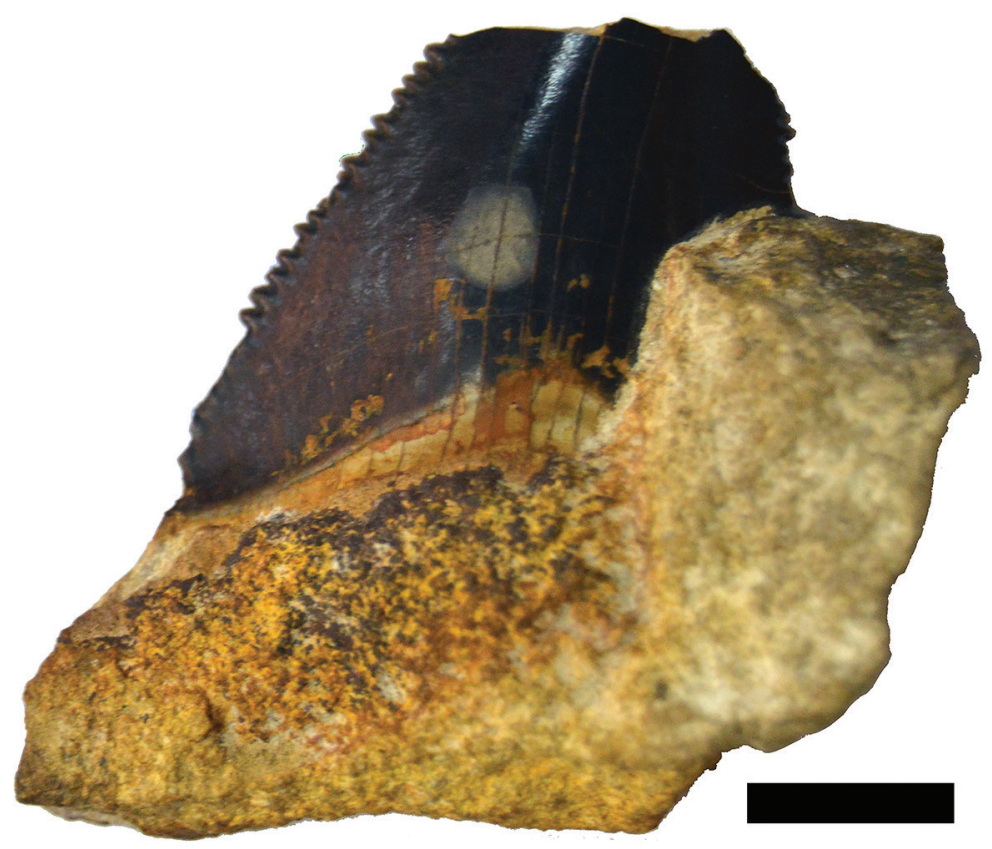

Fig. 3: el CF- 3607 diente anterior superior Otodus (Megaselachus) chubutensis [Ameghino, 1901 a 1902] en vista lingual, escala visual $10 \mathrm{~mm}$.

\section{REFERENCIAS BIBLIOGRÁFICAS}

AGUiLERA, O., 2010: Peces fósiles del Caribe de Venezuela.- 258 págs. Gorham Printing, Washington D.C.

AGUILERA, O. \& RODRIGUES, D., 2004: Gianttoothed White Sharks and Wide-Toothed Mako (Lamnidae) from the Venezuela Neogene: Their Role in the Caribbean, Shallow-Water Fish Assemblage.Caribbean J. Sci. 40(3): 368-382.

CAPPETTA, H., 2012: Chondrichtyes Mesozoic and Cenozoic Elasmobranchii: Teeth.En: SCHULTZE, H. P. (ed.): Handbook of Paleoichthyology, Vol. 3E.- 512 págs. München.
CASE, G., 1980:A Selachian Fauna from the Trent Formation, Lower Miocene of Eastern North Carolina.- Palaeontographica, 171: 75-103.

COOK, T. D., MURRAY, A. M., SIMONS, E. L., ATTIA, Y. S. \& CHATRATH, P., 2010: A Miocene Selachian Fauna from Moghra, Egypt.- Hist. Biol. An Int. J. Paleobiol. 22(1-3): 78-87.

COSTA, S. A., RICHTER, M., DE TOLEDO, P. M. \& MORAES-SANTOS, H. M., 2009: Shark Teeth from Pirabas Formation (Lower Miocene), Northeastern Amazonia, Brazil.- Bol. Mus. Para. Emílio Goeldi. Cienc. Nat. Belém, 4(3): 221-230. 
DENYER, P. \& ARIAS, O., 1991: Estratigrafía de la región central de Costa Rica.- Rev. Geol. Amér. Central, 12: 1-59.

GLÜCKMAN, L. S., 1964: Class Chondrichthyes, Subclass Elasmobranchii.-En: OBRUCHEV, D. V. (ed.): Fundamental of Paleontology.Moscow \& Leningrad (Nauka SSSR), 11: 196-237.

GUZMÁN-GUZMÁN, S.; GONZÁLEZBARBA, G.; SÁNCHEZ-ROMERO, D. Y ROMERO ZÁRATE, A., 2014: Dientes de Elasmobranchii de las formaciones Tuxpan y Encanto del Mioceno de Veracruz. - VIII Congreso latinoamericano de paleontología \& XIII Congreso Nacional de Paleontología, México: 56.

ITURRALDE-VINENT, M., HUBBELL, G. \& ROJAS, R., 1996: Catalogue of Cuban Fossil Elasmobranchii (Paleocene to Pliocene) and Paleogeographic Implications of their Lower to Middle Miocene Occurrence.- J. the Geol. Soc. Jamaica, 31: 7-21.

LAURITO, C. A., CALVO, C., VALERIO, A.L., CALVO, A. \& CHACÓN, R., 2014: Ictiofauna del Mioceno Inferior de la localidad de Pacuare de Tres Equis, Formación Río Banano, provincia de Cartago, Costa Rica, y descripción de un nuevo género y una nueva especie de Scaridae.- Rev. Geol. Amér. Central, 50: 153-192.
MARSILI, S., CARNEVAlE, G., DANESE, E., BIANUCCI, G. \& LANDINI, W., 2007: Early Miocene Vertebrates from Montagna della Maiella, Italy.- An. Paléontologie, 93: 27-66.

MENESINI, E., 1968: Ittiodontoliti Miocenici di Terra d'Otranto (Puglia).- Palaeontographia Italica, 65: 1-61.

MENESINI, E., 1974: Ittiodontoliti delle Formazioni Terziarie dell'Arcipelago Maltese.- Palaeontographia Italica, 67: 121-162.

MORA, C., 1985: Sedimentología y morfología del sur de la península de Nicoya (Provincia de Puntarenas, Costa Rica).148 págs. Univ. de Costa Rica, San José [Tesis Lic.].

PIMIENTO, C., GONZALEZ-BARBA, G., HENDY, A. J. W., JARAMILLO, C., MACFADDEN, B. J., MONTES, C., SUAREZ, S. C. \& SHIPPRITT, M., 2013: Early Miocene Chondrichthyans from the Culebra Formation, Panama: A Window Into Marine Vertebrate Faunas Before Closure the Central American Seaway.- J. South Amer. Earth Sci. 42: 159-170.

PURDY, R., 1998: The Early Miocene Fish Fauna from the Pollack Farm Site, Delaware.- En BENSON. R. N. (ed.): Geology and Paleontology of the Lower 
Miocene Pollack Farm Fossil Site, Delaware.- Delaware Geological Survey Special Publication, Delaware, 21: 133-139.

PURDY, R. W., SCHNEIDER, V. P., APPLEGATE, S. P., MCLELLAN, J. H., MEYER, R. L. \& SLAUGHTER, B. H. 2001. The Neogene Sharks, Rays, and Bony Fishes from Lee Creek Mine, Aurora, North Carolina.- En: RAY, C. E. \& BOHASKA, D. J. (eds): Geology and Paleontology of the Lee Creek Mine, North
Carolina, III.- Smithsonian Contributions to Paleobiol. 90: 71-202.

SAHNI, A. \& MEHROTRA, D. K., 1981: The Elasmobranch Fauna of Coastal Miocene Sediments of Peninsular India.- Biol. Mem., 5: 83-121.

VISAGGI, C. C. \& GODFREY, S. J., 2010: Variation in Composition and Abundance of Miocene Shark Teeth from Calvert Cliffs, Maryland.- J. Vertebrate Paleont. 30: 26-35. 